University of Nebraska - Lincoln

DigitalCommons@University of Nebraska - Lincoln

10-1-1988

\title{
Differential Cross Sections for Secondary Electron Production by 1.5-keV Electrons in Water Vapor
}

K. W. Hollman

University of Nebraska - Lincoln

G. W. Kerby, III

University of Nebraska - Lincoln

M. Eugene Rudd

University of Nebraska - Lincoln, erudd@unl.edu

J. H. Miller

Radiological Physics Section, Pacific Northwest Laboratory, Richland, Washington

S. T. Manson

Georgia State University, Atlanta, Georgia

Follow this and additional works at: https://digitalcommons.unl.edu/physicsrudd

Part of the Physics Commons

Hollman, K. W.; Kerby, III, G. W.; Rudd, M. Eugene; Miller, J. H.; and Manson, S. T., "Differential Cross Sections for Secondary Electron Production by 1.5-keV Electrons in Water Vapor" (1988). M. Eugene Rudd Publications. 5.

https://digitalcommons.unl.edu/physicsrudd/5

This Article is brought to you for free and open access by the Research Papers in Physics and Astronomy at DigitalCommons@University of Nebraska - Lincoln. It has been accepted for inclusion in M. Eugene Rudd Publications by an authorized administrator of DigitalCommons@University of Nebraska - Lincoln. 


\title{
Differential cross sections for secondary electron production by $1.5-\mathrm{keV}$ electrons in water vapor
}

\author{
K. W. Hollman, G. W. Kerby III, and M. E. Rudd \\ Department of Physics and Astronomy, University of Nebraska, Lincoln, Nebraska 68588-0111 \\ J. H. Miller \\ Radiological Physics Section, Pacific Northwest Laboratory, Richland, Washington 99352 \\ S. T. Manson \\ Department of Physics and Astronomy, Georgia State University, Atlanta, Georgia 30303
}

(Received 17 March 1988)

\begin{abstract}
Discrepancies between previous experimental values of differential cross sections for electronimpact ionization of water vapor and recent model calculations have been largely resolved. A new measurement with improved suppression of spurious electrons has removed most of the discrepancy in the midrange of detected electron energies. A second discrepancy at secondary energies just below the primary energy has been explained by a more accurate accounting for electrons scattered at angles between zero and the minimum angle of the experimental apparatus. The improved data show more clearly the oxygen $K$-shell edge in the spectra at small angles and the Bethe ridge at angles up to $90^{\circ}$. The forward peak seen in the earlier data is no longer present.
\end{abstract}

\section{INTRODUCTION}

Measurements of secondary electron production cross sections for electron impact on water vapor as a function of the energy and angle of the ejected electrons were presented by Bolorizadeh and Rudd ${ }^{1}$ over the primary energy range of 50-2000 eV. These doubly differential cross sections (DDCS) were integrated over angle to obtain singly differential cross sections (SDCS). Subsequently, a semiempirical model for the SDCS was developed by Miller et al. ${ }^{2}$ based on an asymptotic expansion of the Born approximation with coefficients evaluated from experimental photoabsorption and proton impact data. Agreement between the model and the experimental data was generally good except in two regions of detected electron energy. One of these was for a final state in which the ejected and scattered electrons were of nearly the same energy. New data have now been taken at a primary energy of $1500 \mathrm{eV}$ which have partially resolved this discrepancy. The new measurements have also shown some features of the electron ejection process more clearly and have removed the forward peak formerly seen at some energies. The second discrepancy in the SDCS occurred for detected electrons close to the primary energy. New calculations have shown that this discrepancy was due to incomplete integration over angles below the smallest measured angle $\left(15^{\circ}\right)$.

\section{APPARATUS}

Since the apparatus used was a modified version of the one described earlier, ${ }^{1}$ only a very brief description will be given. A rotatable electron gun sends a beam through a static gas target. Scattered and secondary electrons at angles of $15^{\circ}-150^{\circ}$ are analyzed electrostatically, detected by a channeltron, and counted. Magnetic shielding inside the vacuum chamber surrounds the entire apparatus. The modification to the detection system is described in Sec. III.

\section{RESULTS AND DISCUSSION}

\section{A. Intermediate-energy data}

The SDCS as a function of detected electron energy falls from a high value near zero energy to a minimum at an intermediate energy and then rises again at energies approaching the impact energy $T$ minus the ionization potential $I$. In the experiment both secondary electrons from the target and scattered primary electrons are detected. Therefore, one must calculate both contributions from any model and add them to compare with experiment.

Miller et $a l .^{2}$ recently developed a model for these cross sections based on an asymptotic expansion of the Born approximation. A significant discrepancy between the model and the experimental data appeared in the vicinity of the minimum in the curve, where the final energies of the two electrons are nearly equal. At this energy the measured cross sections were larger than the calculations by a factor of 2 to 3 for primary energies above 1 $\mathrm{keV}$. Since the cross section at the minimum is approximately 3000 times smaller than the cross section near the primary energy, it was suspected that a small fraction of the numerous high-energy electrons may have found their way into the detector when the electron spectrometer was set to pass the intermediate energies. This would be a particular problem at the forward angles where the highenergy electrons were most numerous. A preliminary run with better suppression of stray electrons confirmed this idea. 
New measurements were therefore made at all angles and detected energies for a primary energy of $1500 \mathrm{eV}$ after adding a biased slit between the exit slit of the parallel-plate analyzer and the cone of the channeltron detector. A grid at that location was first tried but secondary electron production from electrons striking the grid actually made the problem worse. As a test of the effectiveness of this suppressor, 300-eV electrons were sent through helium gas and the spectrum of the electrons scattered at $15^{\circ}$ was measured with different potentials on this electrode. The results are shown in Fig. 1. The curve with the electrode at zero potential should be similar to what would be obtained with no additional suppression, thus approximating the conditions in the previous work. When the analyzer potential is above the energy of the elastic peak, say at $325 \mathrm{~V}$, the count rate should ideally be zero since no electrons of that energy are possible. However, the detector records an appreciable count. Even though the count is 2 to 3 orders of magnitude lower than the peak, when measuring very small cross sections even a small number of spurious counts can change the measured values by an appreciable amount. However, as shown in Fig. 1, when a negative potential equal to $\frac{1}{4}$ or $\frac{1}{2}$ of the analyzer back plate potential is applied to the suppressor, the high-energy tail of the curve is markedly reduced.

Therefore, data were taken with the suppressor electrode held at one-half the analyzer back plate potential $V_{A}$. In our analyzer the back plate potential is equal to the energy of the electrons passed by the analyzer so the suppressor has little effect on electrons of the proper energy to pass the analyzer. The new data were normalized by comparison with the earlier measurements ${ }^{1}$ in a region of the secondary electron spectrum $(20-60 \mathrm{eV})$ where the effect of the added slit should be negligible. This comparison indicated that only a $2-3 \%$ adjustment was needed in the overall efficiency. However, the resulting cross sections measured with the biased slit were significantly

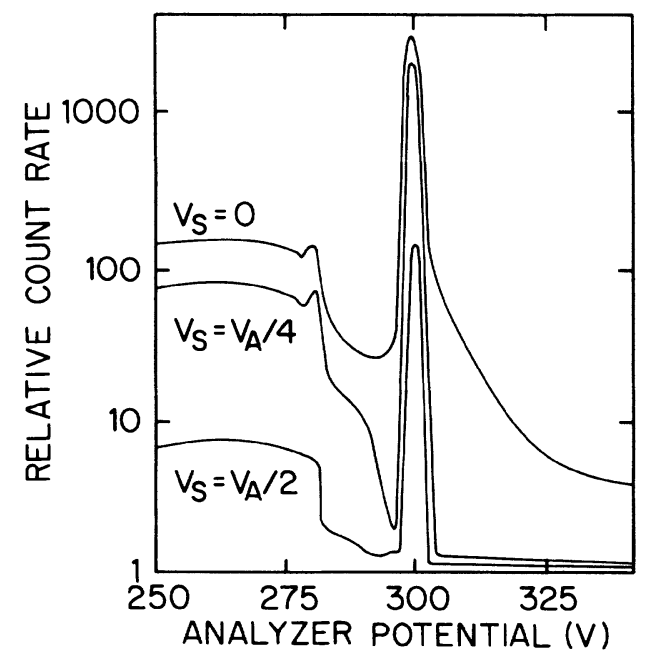

FIG. 1. Relative count rate at $15^{\circ}$ for electrons detected near the elastic peak for $300-\mathrm{eV}$ electrons on helium gas. Results using three different suppressor potentials are shown. lower at the minimum of the curve especially at small angles. Comparisons of the original and revised SDCS are shown in Fig. 2 along with the values calculated from the model. Although there is a residual discrepancy, at its greatest it is a factor of 1.38. This agreement is felt to be acceptable considering the difficulty of measuring very small numbers of electrons at one energy in the presence of much larger numbers of electrons at higher (and lower) energy. Multiple ionization also would be expected to populate that region of the spectrum and may account for part of the residual discrepancy.

Another result of the improved suppression of spurious electrons is the uncovering of some features which were previously obscured. These will be discussed in later sections.

\section{B. High-energy data}

A second discrepancy between the original data and the model occurred at energies just below the primary energy. The model predicts a rising curve in that region while the experimental data fall off markedly as shown in Fig. 2. The reason for this has to do with the angular distribution of electrons. As the detected electron energy $W$ approaches the primary energy $T$ the angular distribution is peaked at smaller and smaller angles. This is seen in the experimental data in Fig. 3. Even though the cross section is multiplied by $\sin \theta$ when integrating over angle, there is an increasingly large contribution to the integral at angles below $15^{\circ}$, the smallest angle measured. As in the earlier work, the integration was done by fitting a second-order function to the data between measured points. This did not adequately represent the angular distribution when the peak was between $0^{\circ}$ and $15^{\circ}$.

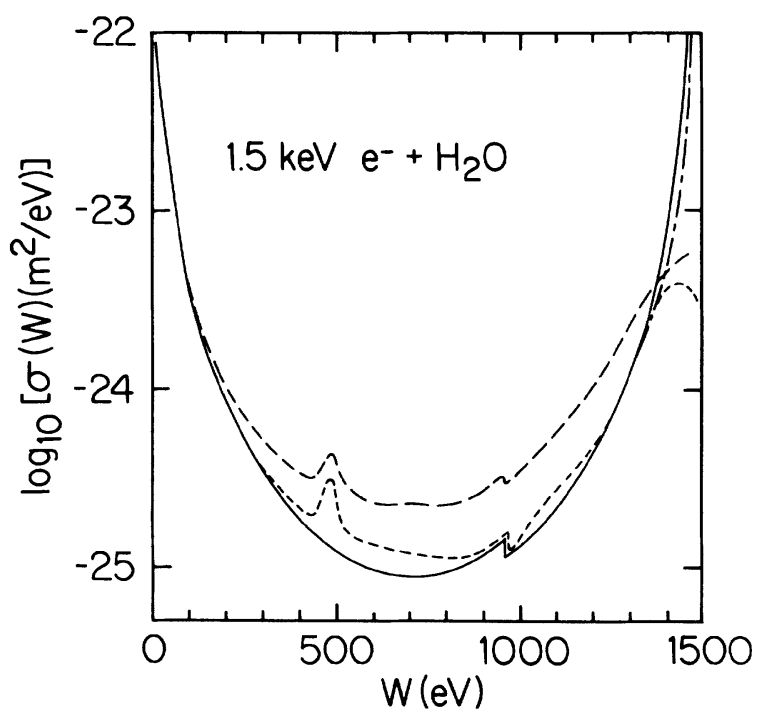

FIG. 2. Energy distribution of electrons, integrated over angles, from $1500 \mathrm{eV} e^{-}+\mathrm{H}_{2} \mathrm{O}$ collisions. Solid line, calculations from the model (Ref. 2); long-dashed line, earlier measurement (Ref. 1); short-dashed line, present data. The dash-dotted line above $1400 \mathrm{eV}$ is the present experimental data after making the small-angle correction (see text). 
An improved estimate of the contribution from smallangle scattering to the SDCS at large $W$ can be made under the assumption that most of the electrons detected in the final state with $W \sim T$ are primary electrons that have undergone a glancing collision with the target molecule. In the first Born approximation, the differential cross section for such a collision can be expressed $\mathrm{as}^{3}$

$$
d\left(\frac{d \theta}{d E}\right)=\frac{4 \pi a_{0}^{2} R^{2}}{T E} \frac{d f(Q, E)}{d E} \frac{d Q}{Q},
$$

where $a_{0}$ is the Bohr radius $(0.529 \AA), R$ is the Rydberg $(13.6 \mathrm{eV}), E$ is the energy lost by the primary electron in the collision, and $d f(Q, E) / d E$ is the generalized oscillator strength (GOS) of the target. $Q$ is a dimensionless variable proportional to the square of the momentum transfer in the collision and related to the scattering angle $\theta$ of the primary electron by

$$
Q=2(T / R)\left[1-(E / 2 T)-(1-E / T)^{1 / 2} \cos \theta\right] .
$$

Although optical oscillator strengths of water have been measured, ${ }^{4}$ the details of the $Q$ dependence of the GOS for this molecule are unknown. From calculations on atomic systems, ${ }^{5}$ we expect the GOS to be independent of momentum transfer at very small values of $Q$. If this is true, then $d Q / Q$ becomes the predominant factor determining the angular dependence of Eq. (1) for smallangle scattering. This factor can be written as $d Q / Q$ $=f(\theta) d \theta \quad$ where $f(\theta)=a \sin \theta /(b-a \cos \theta)$ with

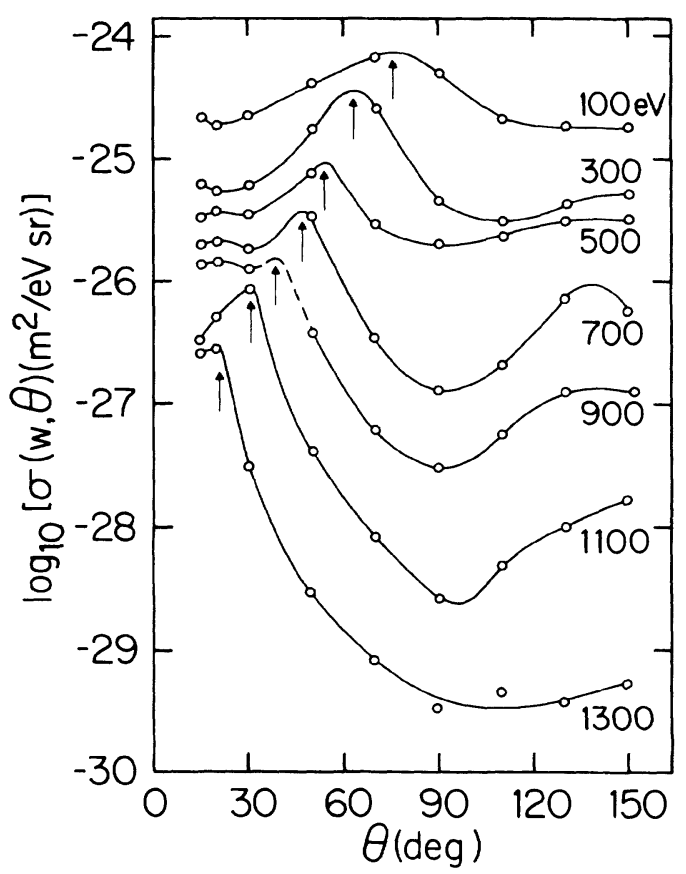

FIG. 3. Angular distributions of secondary electrons from $1500-\mathrm{eV}$ electron impacts on water vapor. The cross-section scale is correct for the $100-\mathrm{eV}$ curve but the other curves have been shifted vertically by arbitrary amounts for clarity. Vertical arrows indicate the predicted positions of the binary encounter peaks. Data were not taken at a sufficient number of angles to show the peak in the $900-\mathrm{eV}$ curve; the dotted line is estimated.
$a=(1-E / T)^{1 / 2}$ and $b=1-E / 2 T . f(\theta)$ is a rapidly varying function which is zero at $\theta=0$ and has a maximum at $\theta=\arccos (a / b)$. The maximum can come at angles smaller than one degree when $E \ll T$. If $E / T$ is small enough to make $Q(\theta=0)$ lie in the region where the GOS is expected to be independent of $Q$, then the optical oscillator strength data for water can be used to estimate the DDCS for glancing collisions of the primary electron. By interpolating between these estimates of the DDCS at small scattering angles and the experimental data at $15^{\circ}$, we can improve the integration over $\theta$ required to obtain the SDCS. Results based on the assumption that the GOS of water is independent of momentum transfer for $\ln (Q)<-2$ are shown in Fig. 2. This method for estimating the contribution to the SDCS from angles less than $15^{\circ}$ gives results that are much closer to those predicted by the semiempirical model ${ }^{2}$ than earlier results ${ }^{1}$ that were based on fitting a quadratic function to the angular dependence of the DDCS.

\section{C. $K$-shell edge}

Data taken with the suppressor show some features of the cross-section dependence clearly that were only barely visible in the earlier data. The oxygen $K$-Auger peak at $500 \mathrm{eV}$ is more pronounced, but most notable is the sharp discontinuity seen at $960 \mathrm{eV}$ in the small angle data shown in Fig. 4. It is most pronounced in the $15^{\circ}$ data where there is a sudden drop of a factor of 2.5 but it is also visible at other angles and in the integrated cross section as shown in Fig. 2. This discontinuity is due to ionization of the $K$ shell of oxygen. Since the $K$-shell binding energy is $540 \mathrm{eV}$, electrons must lose at least that much energy when ionizing that shell. Thus there should be a sharp dropoff at $1500-540=960 \mathrm{eV}$. There should also be such dropoffs for other shells, but in the case of

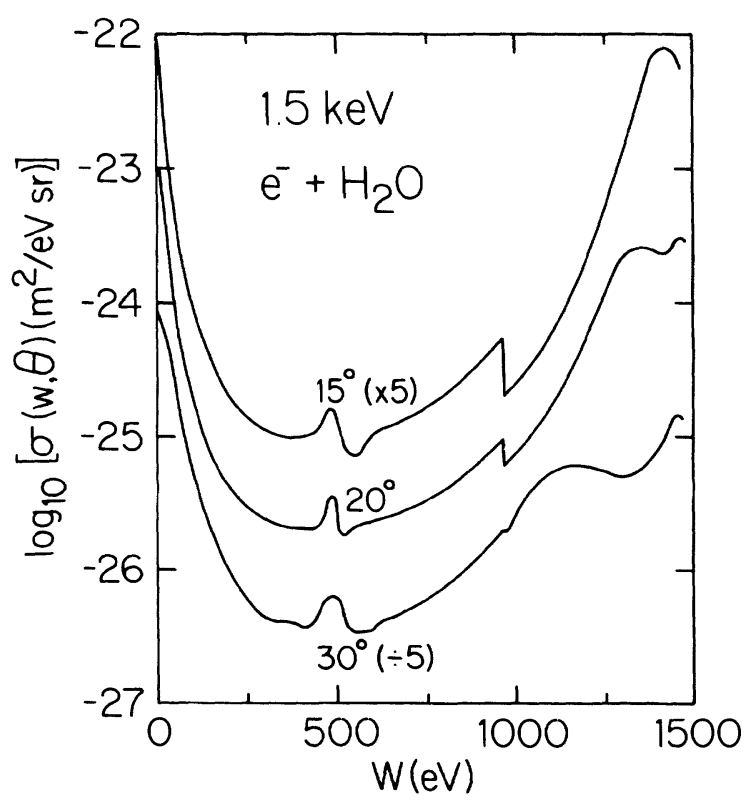

FIG. 4. Experimental energy distributions of electrons ejected at three angles from $1500 \mathrm{eV} e^{-}+\mathrm{H}_{2} \mathrm{O}$ collisions. 


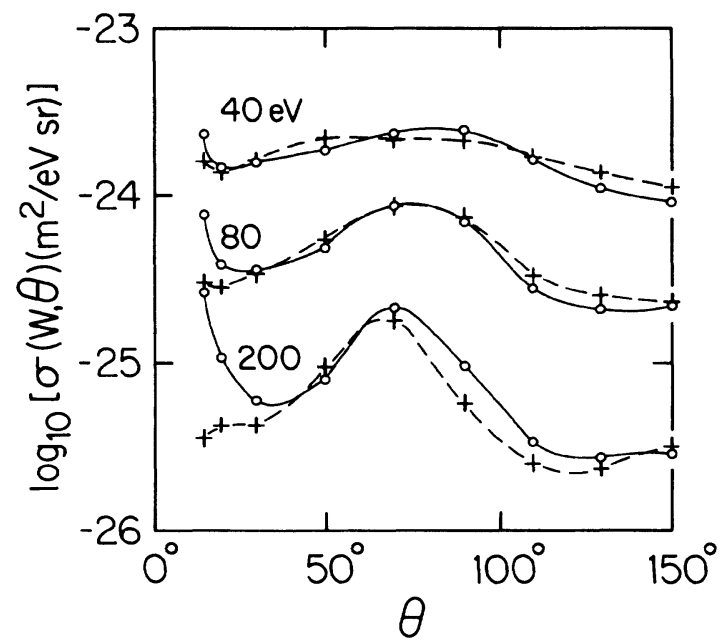

FIG. 5. Angular distributions of secondary electrons from 1500-eV electrons on water vapor. Solid lines are earlier data (Ref. 1). Dashed lines are present data. Earlier peak in the forward direction has been largely eliminated by additional suppression of low-energy electrons.

water vapor the binding energies of all the other shells are only a few tens of $\mathrm{eV}$ and therefore are too close to the primary energy to be resolved.

\section{The Bethe ridge}

Another set of features that show clearly in this data are the binary encounter peaks that constitute the Bethe ridge. A maximum in the angular distribution for each detected energy is observed at the angle for which an initially free, stationary electron of the given final energy would be seen after the collision. These angles, which are easily calculated from simple momentum and energy considerations, are indicated by the vertical arrows in Fig. 3. These maxima have been seen before ${ }^{6,7}$ but not over as wide a range of angles.

\section{E. Peak in the forward direction}

A peak in the angular distribution at zero angle was seen by $\mathrm{Oda}^{8}$ and by Ehrhardt et al. ${ }^{9}$ and interpreted by Tahira and $\mathrm{Oda}^{10}$ as being due to exchange. However, Oda and Nishimura ${ }^{11}$ found that they were able to elimi- nate the peak by adding a retarding potential between their analyzer and detector and improving their electron gun optics. Such a forward peak was also seen in the earlier work from this laboratory ${ }^{1}$ although it was noted that it was possible to suppress the peak with a properly biased electrode. In the present work the suppressor, which was similar to that of Oda and Nishimura, also eliminated the forward peak as shown in Fig. 5.

\section{FINAL REMARKS}

The experimental work reported above emphasizes several problems endemic to electron spectroscopy in atomic collisions, along with the methodology to solve them. The counting of small numbers of electrons at one energy in the presence of much larger numbers of electrons at other energies was one of these problems. The second involved integration of the DDCS over angles to obtain a SDCS; in such a case care must be taken to be sure that the peak at some angles in the DDCS is included in the angular range measured. If not, theoretical ideas may be used to extrapolate the measured cross sections into the range where measurements were not made.

The quality of the present experimental data, as compared to our previous data, is clearly demonstrated in Fig. 2 in the vicinity of the $K$ edge. Simple conservation of energy arguments tell us that the $K$ edge must be there, and perusal of Fig. 2 shows how much more clearly resolved it is in the new data. The same is clearly true of the Auger peak, also seen in Fig. 2. Thus we see that inspection of the inner-shell edges gives insight into the quality and resolution of the experimental data.

Finally, this work shows the importance and utility of theory and experiment proceeding interactively. Without the comparison with theory, we might never have known of the difficulties in the previous experimental data. We cannot overemphasize this point.

\section{ACKNOWLEDGMENTS}

The experimental work reported here was supported by the National Science Foundation under Grants No. PHY 8401328 and No. PHY 8701905. One of us (J.H.M.) acknowledges support by the U.S. Department of Energy Office of Health and Environmental Research under Contract No. DE-AC06-76RLO 1830. Another (S.T.M.) acknowledges support by the Army Research Office under Contract No. DAAL03-86-K-0085.
${ }^{1}$ M. A. Bolorizadeh and M. E. Rudd, Phys. Rev. A 33, 882 (1986).

${ }^{2}$ J. H. Miller, W. E. Wilson, S. T. Manson, and M. E. Rudd, J. Chem. Phys. 86, 157 (1987).

${ }^{3}$ M. Inokuti, Rev. Mod. Phys. 43, 297 (1971).

${ }^{4}$ C. E. Brion and F. Carnovale, Chem. Phys. 100, 291 (1985).

${ }^{5}$ S. T. Manson, Phys. Rev. A 6, 1013 (1972).

${ }^{6}$ C. B. O. Mohr and F. H. Nicoll, Proc. R. Soc. London, Ser. A 144, 596 (1934).

${ }^{7}$ Eg, C. B. Opal, W. K. Peterson, and E. C. Beaty, J. Chem.
Phys. 55, 4100 (1971).

${ }^{8}$ Nobuo Oda, Radiat. Res. 64, 80 (1975).

${ }^{9}$ H. Ehrhardt, K. H. Hesselbacher, K. Jung, M. Schulz, T. Takaat, and K. Willmann, Z. Phys. 244, 254 (1971).

${ }^{10}$ Sadamu Tahira and Nobuo Oda, J. Phys. Soc. Jpn. 35, 582 (1973).

${ }^{11}$ N. Oda and F. Nishimura, in Abstracts of the Tenth International Conference on the Physics of Electronic and Atomic Collisions, edited by M. Barat and J. Reinhardt (Commissariat a L'Energie Atomique, Paris, 1977), p. 362. 